\title{
Fuzzy Stability of a General Quadratic Functional Equation
}

\author{
Sun Sook Jin and Yang-Hi Lee \\ Department of Mathematics Education, Gongju National University of Education, Gongju 314-711, Republic of Korea
}

Correspondence should be addressed to Yang-Hi Lee, yanghi2@hanmail.net

Received 15 March 2011; Accepted 17 May 2011

Academic Editor: Rustom M. Mamlook

Copyright ( $\odot 2011$ S. S. Jin and Y.-H. Lee. This is an open access article distributed under the Creative Commons Attribution License, which permits unrestricted use, distribution, and reproduction in any medium, provided the original work is properly cited.

We investigate a fuzzy version of stability for the functional equation $f(x+y+z)+f(x-y)+f(x-z)-f(x-y-z)-f(x+$ $y)-f(x+z)=0$ in the sense of M. Mirmostafaee and M. S. Moslehian.

\section{Introduction and Preliminaries}

A classical question in the theory of functional equations is "when is it true that a mapping, which approximately satisfies a functional equation, must be somehow close to an exact solution of the equation?". Such a problem, called a stability problem of the functional equation, was formulated by Ulam [1] in 1940. In the next year, Hyers [2] gave a partial solution of Ulam's problem for the case of approximate additive mappings. Subsequently, his result was generalized by Aoki [3] for additive mappings, and by Rassias [4] for linear mappings, to consider the stability problem with unbounded Cauchy differences. During the last decades, the stability problems of functional equations have been extensively investigated by a number of mathematicians, see [5-15].

In 1984, Katsaras [16] defined a fuzzy norm on a linear space to construct a fuzzy structure on the space. Since then, some mathematicians have introduced several types of fuzzy norm in different points of view. In particular, Bag and Samanta [17], following Cheng and Mordeson [18], gave an idea of a fuzzy norm in such a manner that the corresponding fuzzy metric is of Kramosil and Michálek type [19]. In 2008, Mirmostafaee and Moslehian [20] obtained a fuzzy version of stability for the Cauchy functional equation:

$$
f(x+y)-f(x)-f(y)=0 .
$$

In the same year, they [21] proved a fuzzy version of stability for the quadratic functional equation:

$$
f(x+y)+f(x-y)-2 f(x)-2 f(y)=0 .
$$

We call a solution of (1) an additive map, and a solution of (2) is called a quadratic map. Now we consider the functional equation:

$$
\begin{aligned}
& f(x+y+z)+f(x-y)+f(x-z) \\
& \quad-f(x-y-z)-f(x+y)-f(x+z)=0,
\end{aligned}
$$

which is called a general quadratic functional equation. We call a solution of (3) a general quadratic function. Recently, Kim [22] and Jun and Kim [23] obtained a stability of the functional equation (3) by taking and composing an additive map $A$ and a quadratic map $Q$ to prove the existence of a general quadratic function $F$ which is close to the given function $f$. In their processing, $A$ is approximate to the odd part $(f(x)-f(-x)) / 2$ of $f$, and $Q$ is close to the even part $(f(x)+f(-x)) / 2-f(0)$ of it, respectively.

In this paper, we get a general stability result of the general quadratic functional equation (3) in the fuzzy normed linear space. To do it, we introduce a Cauchy sequence $\left\{J_{n} f(x)\right\}$, starting from a given function $f$, which converges to the desired function $F$ in the fuzzy sense. As we mentioned before, in previous studies of stability problem of (3), they attempted to get stability theorems by handling the odd and even part of $f$, respectively. According to our proposal in this paper, we can take the desired approximate solution $F$ at once. Therefore, this idea is a refinement with respect to the simplicity of the proof. 


\section{Fuzzy Stability of the Functional Equation (3)}

We use the definition of a fuzzy normed space given in [17] to exhibit a reasonable fuzzy version of stability for the general quadratic functional equation in the fuzzy normed linear space.

Definition 1 (see [17]). Let $X$ be a real linear space. A function $N: X \times \mathbb{R} \rightarrow[0,1]$ (the so-called fuzzy subset) is said to be a fuzzy norm on $X$ if for all $x, y \in X$ and all $s, t \in \mathbb{R}$,

(N1) $N(x, c)=0$ for $c \leq 0$,

(N2) $x=0$ if and only if $N(x, c)=1$ for all $c>0$,

(N3) $N(c x, t)=N(x, t /|c|)$ if $c \neq 0$,

(N4) $N(x+y, s+t) \geq \min \{N(x, s), N(y, t)\}$,

(N5) $N(x, \cdot)$ is a nondecreasing function on $\mathbb{R}$, and $\lim _{t \rightarrow \infty} N(x, t)=1$.

The pair $(X, N)$ is called a fuzzy normed linear space. Let $(X, N)$ be a fuzzy normed linear space. Let $\left\{x_{n}\right\}$ be a sequence in $X$. Then $\left\{x_{n}\right\}$ is said to be convergent if there exists $x \in X$ such that $\lim _{n \rightarrow \infty} N\left(x_{n}-x, t\right)=1$, for all $t>0$. In this case, $x$ is called the limit of the sequence $\left\{x_{n}\right\}$ and we denote it by $N-\lim _{n \rightarrow \infty} x_{n}=x$. A sequence $\left\{x_{n}\right\}$, in $X$ is called Cauchy if for each $\varepsilon>0$ and each $t>0$, there exists $n_{0}$ such that for all $n \geq n_{0}$ and all $p>0$, we have $N\left(\mathrm{x}_{n+p}-x_{n}, t\right)>1-\varepsilon$. It is known that every convergent sequence in a fuzzy normed space is Cauchy. If each Cauchy sequence is convergent, then the fuzzy norm is said to be complete, and the fuzzy normed space is called a fuzzy Banach space.

Let $(X, N)$ be a fuzzy normed space, and let $\left(Y, N^{\prime}\right)$ be a fuzzy Banach space. For a given mapping $f: X \rightarrow Y$, we use the abbreviation

$$
\begin{aligned}
D f(x, y, z):= & f(x+y+z)+f(x-y)+f(x-z) \\
& -f(x-y-z)-f(x+y)-f(x+z),
\end{aligned}
$$

for all $x, y, z \in X$. Recall $D f \equiv 0$ means that $f$ is a general quadratic function. For given $q>0$, the function $f$ is called a fuzzy q-almost general quadratic function, if

$$
\begin{aligned}
& N^{\prime}(D f(x, y, z), r+s+t) \\
& \quad \geq \min \left\{N\left(x, r^{q}\right), N\left(y, s^{q}\right), N\left(z, t^{q}\right)\right\},
\end{aligned}
$$

for all $x, y, z \in X$ and $r, s, t \in[0, \infty)$. Now we get the general stability result in the fuzzy normed linear setting.

Theorem 1. Let $q$ be a positive real number with $q \neq 1 / 2,1$. And let $f$ be a fuzzy q-almost general quadratic function froma fuzzy normed space $(X, N)$ into a fuzzy Banach space $\left(Y, N^{\prime}\right)$. Then there is a unique general quadratic function $F: X \rightarrow Y$ such that

$$
\begin{aligned}
& N^{\prime}(F(x)-f(x), t) \\
& \quad \geq \sup _{t^{\prime}<t} N\left(x, t^{\prime q} /\left(\frac{5+2^{p}}{2^{p}\left|4-2^{p}\right|}+\frac{2 \cdot 2^{p}+7}{2 \mid 2-2^{p \mid}}\right)^{q}\right),
\end{aligned}
$$

for all $x \in X$ and $t>0$, where $p=1 / q$.

Proof. We will prove the theorem in three cases, $q>1,1 / 2<$ $q<1$, and $0<q<1 / 2$.

Case 1. Let $q>1$. We define the function $J_{n} f: X \rightarrow Y$ by

$$
\begin{aligned}
J_{n} f(x):= & \frac{f\left(2^{n} x\right)+f\left(-2^{n} x\right)-2 f(0)}{2 \cdot 4^{n}}+\frac{f\left(2^{n} x\right)-f\left(-2^{n} x\right)}{2^{n+1}} \\
& +f(0),
\end{aligned}
$$

for all $x \in X$. Notice that $J_{0} f(x)=f(x), J_{j} f(0)=f(0)$, and

$$
\begin{aligned}
J_{j} f(x) & -J_{j+1} f(x) \\
= & -\frac{D f\left(2^{j-1} x, 2^{j-1} x, 2^{j} x\right)}{2 \cdot 4^{j+1}}-\frac{D f\left(2^{j-1} x, 2^{j-1} x, 2^{j-1} x\right)}{2 \cdot 4^{j+1}} \\
& -\frac{D f\left(-2^{j-1} x,-2^{j-1} x,-2^{j} x\right)}{2 \cdot 4^{j+1}} \\
& -\frac{D f\left(-2^{j-1} x,-2^{j-1} x,-2^{j-1} x\right)}{2 \cdot 4^{j+1}} \\
& +\frac{D f\left(2^{j+1} x, 2^{j} x, 2^{j} x\right)}{2^{j+2}}-\frac{D f\left(2^{j} x, 2^{j+1} x, 2^{j} x\right)}{2^{j+2}} \\
& +\frac{D f\left(2^{j} x, 2^{j} x, 2^{j} x\right)}{2^{j+2}},
\end{aligned}
$$

for all $x \in X$ and $j \geq 0$. Together with (N3), (N4) and (5), this equation implies that if $n+m>m \geq 0$ then

$$
\begin{aligned}
& N^{\prime}\left(J_{m} f(x)-J_{n+m} f(x), \sum_{j=m}^{n+m-1}\left(\frac{5+2^{p}}{4 \cdot 2^{p}}\left(\frac{2^{p}}{4}\right)^{j}+\left(\frac{2^{p}}{2}+\frac{7}{4}\right)\left(\frac{2^{p}}{2}\right)^{j}\right) t^{p}\right) \\
& \geq N^{\prime}\left(\sum_{j=m}^{n+m-1}\left(J_{j} f(x)-J_{j+1} f(x)\right), \sum_{j=m}^{n+m-1}\left(\frac{5+2^{p}}{4 \cdot 2^{p}}\left(\frac{2^{p}}{4}\right)^{j}+\left(\frac{2^{p}}{2}+\frac{7}{4}\right)\left(\frac{2^{p}}{2}\right)^{j}\right) t^{p}\right), \\
& \geq \min \bigcup_{j=m}^{n+m-1}\left\{N^{\prime}\left(J_{j} f(x)-J_{j+1} f(x),\left(\frac{5+2^{p}}{4 \cdot 2^{p}}\left(\frac{2^{p}}{4}\right)^{j}+\left(\frac{2^{p}}{2}+\frac{7}{4}\right)\left(\frac{2^{p}}{2}\right)^{j}\right) t^{p}\right)\right\},
\end{aligned}
$$




$$
\begin{aligned}
\geq \min \bigcup_{j=m}^{n+m-1}\left\{N^{\prime}\left(-\frac{D f\left(2^{j-1} x, 2^{j-1} x, 2^{j} x\right)}{2 \cdot 4^{j+1}}, \frac{\left(2^{j p}+2 \cdot 2^{(j-1) p}\right) t^{p}}{2 \cdot 4^{j+1}}\right),\right. \\
N^{\prime}\left(-\frac{D f\left(2^{j-1} x, 2^{j-1} x, 2^{j-1} x\right)}{2 \cdot 4^{j+1}}, \frac{3 \cdot 2^{(j-1) p} t^{p}}{2 \cdot 4^{j+1}}\right), \\
N^{\prime}\left(-\frac{D f\left(-2^{j-1} x,-2^{j-1} x,-2^{j} x\right)}{2 \cdot 4^{j+1}}, \frac{\left(2^{j p}+2 \cdot 2^{(j-1) p}\right) t^{p}}{2 \cdot 4^{j+1}}\right), \\
N^{\prime}\left(-\frac{D f\left(-2^{j-1} x,-2^{j-1} x,-2^{j-1} x\right)}{2 \cdot 4^{j+1}}, \frac{3 \cdot 2^{(j-1) p} t^{p}}{2 \cdot 4^{j+1}}\right), \\
N^{\prime}\left(-\frac{D f\left(2^{j+1} x, 2^{j} x, 2^{j} x\right)}{2^{j+2}}, \frac{\left(2 \cdot 2^{j p}+2^{(j+1) p}\right) t^{p}}{2^{j+2}}\right), \\
\left.N^{\prime}\left(\frac{D f\left(2^{j} x, 2^{j+1} x, 2^{j} x\right)}{2^{j+2}}, \frac{\left(2 \cdot 2^{j p} x, 2^{j} x\right)}{2^{j+2}}, \frac{\left.3 \cdot 2^{j+1} p\right) t^{p}}{2^{j+2}}\right)\right\} \\
\geq \min \bigcup_{j=m}^{n+m-1}\left\{N\left(2^{j} x, 2^{j} t\right), N\left(2^{j-1} x, 2^{j-1} t\right), N\left(2^{j+1} x, 2^{j+1} t\right)\right\}, \\
=N(x, t),
\end{aligned}
$$

for all $x \in X$ and $t>0$. Let $\varepsilon>0$ be given. Since $\lim _{t \rightarrow \infty} N(x, t)=1$, there is $t_{0}>0$ such that

$$
N\left(x, t_{0}\right) \geq 1-\varepsilon .
$$

We observe that for some $\tilde{t}>t_{0}$, the series $\sum_{j=0}^{\infty}\left(\left(5+2^{p}\right) / 4\right.$. $2^{p}\left(2^{p / 4}\right)^{j}+\left(\left(2^{p} / 2\right)+(7 / 4)\right)\left(2^{p} / 2^{j}\right) \tilde{t}^{p}$ converges for $p=1 / q<$ 1. It guarantees that, for an arbitrary given $c>0$, there exists $n_{0} \geq 0$ such that

$$
\sum_{j=m}^{n+m-1}\left(\frac{5+2^{p}}{4 \cdot 2^{p}}\left(\frac{2^{p}}{4}\right)^{j}+\left(\frac{2^{p}}{2}+\frac{7}{4}\right)\left(\frac{2^{p}}{2}\right)^{j}\right) \tilde{t}^{p}<c,
$$

for each $m \geq n_{0}$ and $n>0$. Together with (N5) and (9), this implies that

$$
\begin{aligned}
& N^{\prime}\left(J_{m} f(x)-J_{n+m} f(x), c\right) \\
& \geq N^{\prime}\left(J_{m} f(x)-J_{n+m} f(x),\right. \\
& \left.\quad \sum_{j=m}^{n+m-1}\left(\frac{5+2^{p}}{4 \cdot 2^{p}}\left(\frac{2^{p}}{4}\right)^{j}+\left(\frac{2^{p}}{2}+\frac{7}{4}\right)\left(\frac{2^{p}}{2}\right)^{j}\right) \tilde{t}^{p}\right), \\
& \geq N(x, \tilde{t}) \geq N\left(x, t_{0}\right) \geq 1-\varepsilon,
\end{aligned}
$$

for all $x \in X$. Hence $\left\{J_{n} f(x)\right\}$ is a Cauchy sequence in the fuzzy Banach space $\left(Y, N^{\prime}\right)$. And so we can define a mapping $F: X \rightarrow Y$ by

$$
F(x):=N^{\prime}-\lim _{n \rightarrow \infty} J_{n} f(x)
$$

for all $x \in X$. Moreover, if we put $m=0$ in (9), we have

$$
\begin{aligned}
& N^{\prime}\left(f(x)-J_{n} f(x), t\right) \\
& \quad \geq N\left(x, t^{q} /\left(\sum_{j=0}^{n-1}\left(\frac{5+2^{p}}{4 \cdot 2^{p}}\left(\frac{2^{p}}{4}\right)^{j}+\left(\frac{2^{p}}{2}+\frac{7}{4}\right)\left(\frac{2^{p}}{2}\right)^{j}\right)\right)^{q}\right),
\end{aligned}
$$

for all $x \in X$. Next we will show that $F$ is a desired general quadratic function. Using (N4), we have

$$
\begin{aligned}
& N^{\prime}(D F(x, y, z), t) \\
& \geq \min \left\{N^{\prime}\left(\left(F-J_{n} f\right)(x+y+z), \frac{t}{24}\right),\right. \\
& \quad N^{\prime}\left(\left(F-J_{n} f\right)(x-y), \frac{t}{24}\right), \\
& \quad N^{\prime}\left(\left(F-J_{n} f\right)(x-z), \frac{t}{24}\right),
\end{aligned}
$$




$$
\begin{aligned}
& N^{\prime}\left(\left(J_{n} f-F\right)(x-y-z), \frac{t}{24}\right), \\
& N^{\prime}\left(\left(J_{n} f-F\right)(x+y), \frac{t}{24}\right), \\
& N^{\prime}\left(\left(J_{n} f-F\right)(x+z), \frac{t}{24}\right), \\
& \left.N^{\prime}\left(D J_{n} f(x, y, z), \frac{3 t}{4}\right)\right\},
\end{aligned}
$$

for all $x, y, z \in X$ and $n \in \mathbb{N}$. The first six terms on the right hand side of (15) tend to 1 as $n \rightarrow \infty$ by the definition of $F$ and (N2), and the last term holds

$$
\begin{aligned}
N^{\prime}\left(D J_{n} f(x, y, z), \frac{3 t}{4}\right) \\
\geq \min \left\{N^{\prime}\left(\frac{D f\left(2^{n} x, 2^{n} y, 2^{n} z\right)}{2 \cdot 4^{n}}, \frac{3 t}{16}\right),\right. \\
N^{\prime}\left(\frac{D f\left(-2^{n} x,-2^{n} y,-2^{n} z\right)}{2 \cdot 4^{n}}, \frac{3 t}{16}\right), \\
N^{\prime}\left(\frac{D f\left(2^{n} x, 2^{n} y, 2^{n} z\right)}{2 \cdot 2^{n}}, \frac{3 t}{16}\right), \\
\left.N^{\prime}\left(\frac{D f\left(-2^{n} x,-2^{n} y,-2^{n} z\right)}{2 \cdot 2^{n}}, \frac{3 t}{16}\right)\right\},
\end{aligned}
$$

for all $x, y, z \in X$. By (N3) and (5), we obtain

$$
\begin{aligned}
& N^{\prime}\left(\frac{D f\left( \pm 2^{n} x, \pm 2^{n} y, \pm 2^{n} z\right)}{2 \cdot 4^{n}}, \frac{3 t}{16}\right) \\
& \quad=N^{\prime}\left(D f\left( \pm 2^{n} x, \pm 2^{n} y, \pm 2^{n} z\right), \frac{3 \cdot 4^{n} t}{8}\right),
\end{aligned}
$$

$$
\begin{aligned}
& \geq \min \left\{N\left( \pm 2^{n} x,\left(\frac{4^{n} t}{8}\right)^{q}\right), N\left( \pm 2^{n} y,\left(\frac{4^{n} t}{8}\right)^{q}\right),\right. \\
& \left.N\left( \pm 2^{n} z,\left(\frac{4^{n} t}{8}\right)^{q}\right)\right\}, \\
& \geq \min \left\{N\left(x, 2^{(2 q-1) n-3 q} t^{q}\right), N\left(y, 2^{(2 q-1) n-3 q} t^{q}\right),\right. \\
& N^{\prime}\left(\frac{D f\left( \pm 2^{n} x, \pm 2^{n} y, \pm 2^{n} z\right)}{2 \cdot 2^{n}}, \frac{3 t}{16}\right), \\
& \geq \min \left\{N\left(x, 2^{(2 q-1) n-3 q} t^{q}\right)\right\}, \\
& \left.N\left(z, 2^{(q-1) n-3 q} t^{q}\right)\right\},
\end{aligned}
$$

for all $x, y, z \in X$ and $n \in \mathbb{N}$. Since $q>1$, together with (N5), we can deduce that the last term of (15) also tends to 1 as $n \rightarrow \infty$. It follows from (15) that

$$
N^{\prime}(D F(x, y, z), t)=1,
$$

for all $x, y, z \in X$ and $t>0$. By (N2), it leads us to prove that $F$ is a general quadratic function.

For an arbitrary fixed $x \in X$ and $t>0$, choose $0<\varepsilon<1$ and $0<t^{\prime}<t$. Since $F$ is the limit of $\left\{J_{n} f(x)\right\}$, there is $n \in \mathbb{N}$ such that $N^{\prime}\left(F(x)-J_{n} f(x), t-t^{\prime}\right) \geq 1-\varepsilon$. By (14), we have

$$
\begin{aligned}
N^{\prime}(F(x)-f(x), t) & \geq \min \left\{N^{\prime}\left(F(x)-J_{n} f(x), t-t^{\prime}\right), N^{\prime}\left(J_{n} f(x)-f(x), t^{\prime}\right)\right\}, \\
& \geq \min \left\{1-\varepsilon, N\left(x, t^{\prime q} /\left(\sum_{j=0}^{n-1}\left(\frac{5+2^{p}}{4 \cdot 2^{p}}\left(\frac{2^{p}}{4}\right)^{j}+\left(\frac{2^{p}}{2}+\frac{7}{4}\right)\left(\frac{2^{p}}{2}\right)^{j}\right)\right)^{q}\right)\right\}, \\
& \geq \min \left\{1-\varepsilon, N\left(x, t^{\prime q} /\left(\frac{5+2^{p}}{2^{p} \mid 4-2^{p \mid}}+\frac{2 \cdot 2^{p}+7}{2 \mid 2^{p}-2^{p \mid}}\right)^{q}\right)\right\} .
\end{aligned}
$$

Because $0<\varepsilon<1$ is arbitrary, we get the inequality (6) in this case.

Finally, to prove the uniqueness of $F$, let $F^{\prime}: X \rightarrow Y$ be another general quadratic function satisfying (6). Then by (8), we get

$$
\begin{gathered}
F(x)-J_{n} F(x)=\sum_{j=0}^{n-1}\left(J_{j} F(x)-J_{j+1} F(x)\right)=0, \\
F^{\prime}(x)-J_{n} F^{\prime}(x)=\sum_{j=0}^{n-1}\left(J_{j} F^{\prime}(x)-J_{j+1} F^{\prime}(x)\right)=0,
\end{gathered}
$$


for all $x \in X$ and $n \in \mathbb{N}$. Together with (N4) and (6), this implies that

$$
\begin{aligned}
& N^{\prime}\left(F(x)-F^{\prime}(x), t\right) \\
& =N^{\prime}\left(J_{n} F(x)-J_{n} F^{\prime}(x), t\right), \\
& \geq \min \left\{N^{\prime}\left(J_{n} F(x)-J_{n} f(x), \frac{t}{2}\right),\right. \\
& \left.\quad N^{\prime}\left(J_{n} f(x)-J_{n} F^{\prime}(x), \frac{t}{2}\right)\right\}, \\
& \geq \min \left\{N^{\prime}\left(\frac{(F-f)\left(2^{n} x\right)}{2 \cdot 4^{n}}, \frac{t}{8}\right), N^{\prime}\left(\frac{\left(f-F^{\prime}\right)\left(2^{n} x\right)}{2 \cdot 4^{n}}, \frac{t}{8}\right),\right. \\
& \quad N^{\prime}\left(\frac{(F-f)\left(-2^{n} x\right)}{2 \cdot 4^{n}}, \frac{t}{8}\right), N^{\prime}\left(\frac{\left(f-F^{\prime}\right)\left(-2^{n} x\right)}{2 \cdot 4^{n}}, \frac{t}{8}\right), \\
& \left.\quad N^{\prime}\left(\frac{(F-f)\left(-2^{n} x\right)}{2 \cdot 2^{n}}, \frac{t}{8}\right), N^{\prime}\left(\frac{\left(f-F^{\prime}\right)\left(-2^{n} x\right)}{2 \cdot 2^{n}}, \frac{t}{8}\right)\right\}, \\
& \geq \sup _{t^{\prime}<t} N\left(x, 2^{(q-1) n-2 q} t^{\prime q} /\left(\frac{t}{8}\right), N^{\prime}\left(\frac{\left(f-F^{\prime}\right)\left(2^{n} x\right)}{2 \cdot 2^{n}}, \frac{t}{8}\right),\right.
\end{aligned}
$$

for all $x \in X$ and $n \in \mathbb{N}$. Observe that, for $q=1 / p>1$, the last term of the above inequality tends to 1 as $n \rightarrow \infty$ by (N5). This implies that $N^{\prime}\left(F(x)-F^{\prime}(x), t\right)=1$, and so we get

$$
F(x)=F^{\prime}(x)
$$

for all $x \in X$ by (N2).
Case 2. Let $1 / 2<q<1$, and let $J_{n} f: X \rightarrow Y$ be a function defined by

$$
\begin{aligned}
J_{n} f(x):= & \frac{f\left(2^{n} x\right)+f\left(-2^{n} x\right)-2 f(0)}{2 \cdot 4^{n}} \\
& +2^{n-1}\left(f\left(\frac{x}{2^{n}}\right)-f\left(-\frac{x}{2^{n}}\right)\right)+f(0),
\end{aligned}
$$

for all $x \in X$. Then we also have $J_{0} f(x)=f(x), J_{j} f(0)=$ $f(0)$, and

$$
\begin{aligned}
J_{j} f(x)-J_{j+1} f(x)= & -\frac{D f\left(2^{j-1} x, 2^{j-1} x, 2^{j} x\right)}{2 \cdot 4^{j+1}} \\
& -\frac{D f\left(2^{j-1} x, 2^{j-1} x, 2^{j-1} x\right)}{2 \cdot 4^{j+1}} \\
& -\frac{D f\left(-2^{j-1} x,-2^{j-1} x,-2^{j} x\right)}{2 \cdot 4^{j+1}} \\
& -\frac{D f\left(-2^{j-1} x,-2^{j-1} x,-2^{j-1} x\right)}{2 \cdot 4^{j+1}} \\
& -2^{j-1} D f\left(\frac{x}{2^{j}}, \frac{x}{2^{j+1}}, \frac{x}{2^{j+1}}\right) \\
& +2^{j-1} D f\left(\frac{x}{2^{j+1}}, \frac{x}{2^{j}}, \frac{x}{2^{j+1}}\right) \\
& -2^{j-1} D f\left(\frac{x}{2^{j+1}}, \frac{x}{2^{j+1}}, \frac{x}{2^{j+1}}\right),
\end{aligned}
$$

$$
\begin{aligned}
& N^{\prime}\left(J_{m} f(x)-J_{n+m} f(x),\right.\left.\sum_{j=m}^{n+m-1}\left(\frac{5+2^{p}}{4 \cdot 2^{p}}\left(\frac{2^{p}}{4}\right)^{j}+\frac{\left(7+2 \cdot 2^{p}\right) 2^{j}}{2 \cdot 2^{(j+1) p}}\right) t^{p}\right), \\
& \geq \min \bigcup_{j=m}^{n+m-1}\left\{N^{\prime}\left(-\frac{D f\left(2^{j-1} x, 2^{j-1} x, 2^{j} x\right)}{2 \cdot 4^{j+1}}, \frac{\left(2^{j p}+2 \cdot 2^{(j-1) p}\right) t^{p}}{2 \cdot 4^{j+1}}\right),\right. \\
& N^{\prime}\left(-\frac{D f\left(2^{j-1} x, 2^{j-1} x, 2^{j-1} x\right)}{2 \cdot 4^{j+1}}, \frac{3 \cdot 2^{(j-1) p} t^{p}}{2 \cdot 4^{j+1}}\right), \\
& N^{\prime}\left(-\frac{D f\left(-2^{j-1} x,-2^{j-1} x,-2^{j} x\right)}{2 \cdot 4^{j+1}}, \frac{\left(2^{j p}+2 \cdot 2^{(j-1) p}\right) t^{p}}{2 \cdot 4^{j+1}}\right), \\
& N^{\prime}\left(-\frac{D f\left(-2^{j-1} x,-2^{j-1} x,-2^{j-1} x\right)}{2 \cdot 4^{j+1}}, \frac{3^{j} \cdot 2^{(j-1) p} t^{p}}{2^{2} \cdot 4^{j+1}}\right), \\
& N^{\prime}\left(-2^{j-1} D f\left(\frac{x}{2^{j}}, \frac{x}{2^{j+1}}, \frac{x}{2^{j+1}}\right), 2^{j-1}\left(\frac{t^{p}}{2^{j p}}+\frac{2 t^{p}}{2^{(j+1) p}}\right)\right),
\end{aligned}
$$




$$
\begin{gathered}
N^{\prime}\left(2^{j-1} D f\left(\frac{x}{2^{j+1}}, \frac{x}{2^{j}}, \frac{x}{2^{j+1}}\right), 2^{j-1}\left(\frac{t^{p}}{2^{j p}}+\frac{2 t^{p}}{2^{(j+1) p}}\right)\right), \\
\left.N^{\prime}\left(-2^{j-1} D f\left(\frac{x}{2^{j+1}}, \frac{x}{2^{j+1}}, \frac{x}{2^{j+1}}\right), \frac{3 \cdot 2^{j-1} t^{p}}{2^{(j+1) p}}\right)\right\}, \\
\geq \min \bigcup_{j=m}^{n+m-1}\left\{N\left(2^{j} x, 2^{j} t\right), N\left(2^{j-1} x, 2^{j-1} t\right), N\left(\frac{x}{2^{j+1}}, \frac{t}{2^{j+1}}\right), N\left(\frac{x}{2^{j}}, \frac{t}{2^{j}}\right)\right\}, \\
=N(x, t) .
\end{gathered}
$$

In the similar argument following (9) of the previous case, we can define the limit $F(x):=N^{\prime}-\lim _{n \rightarrow \infty} J_{n} f(x)$ of the Cauchy sequence $\left\{J_{n} f(x)\right\}$ in the Banach fuzzy space $Y$. Moreover, putting $m=0$ in the above inequality, we have

$$
\begin{aligned}
& N^{\prime}\left(f(x)-J_{n} f(x), t\right) \\
& \quad \geq N\left(x, t^{q} /\left(\sum_{j=0}^{n-1}\left(\frac{5+2^{p}}{4 \cdot 2^{p}}\left(\frac{2^{p}}{4}\right)^{j}+\left(1+\frac{7}{2 \cdot 2^{p}}\right)\left(\frac{2}{2^{p}}\right)^{j}\right)\right)^{q}\right),
\end{aligned}
$$

for each $x \in X$ and $t>0$. To prove that $F$ is a general quadratic function, we have enough to show that the last term of (15) in Case 1 tends to 1 as $n \rightarrow \infty$. By (N3) and (5), we get

$$
\begin{aligned}
& N^{\prime}\left(D J_{n} f(x, y, z), \frac{3 t}{4}\right) \\
& \geq \min \left\{N^{\prime}\left(\frac{D f\left(2^{n} x, 2^{n} y, 2^{n} z\right)}{2 \cdot 4^{n}}, \frac{3 t}{16}\right),\right. \\
& N^{\prime}\left(\frac{D f\left(-2^{n} x,-2^{n} y,-2^{n} z\right)}{2 \cdot 4^{n}}, \frac{3 t}{16}\right) \text {, } \\
& N^{\prime}\left(2^{n-1} D f\left(\frac{x}{2^{n}}, \frac{y}{2^{n}}, \frac{z}{2^{n}}\right), \frac{3 t}{16}\right) \\
& \left.N^{\prime}\left(2^{n-1} D f\left(\frac{-x}{2^{n}}, \frac{-y}{2^{n}}, \frac{-z}{2^{n}}\right), \frac{3 t}{16}\right)\right\}, \\
& \geq \min \left\{N\left(x, 2^{(2 q-1) n-4 q} t^{q}\right), N\left(y, 2^{(2 q-1) n-4 q} t^{q}\right),\right. \\
& N\left(z, 2^{(2 q-1) n-4 q} t^{q}\right), N\left(x, 2^{(1-q) n-4 q} t^{q}\right), \\
& \left.N\left(y, 2^{(1-q) n-4 q} t^{q}\right), N\left(z, 2^{(1-q) n-4 q} t^{q}\right)\right\},
\end{aligned}
$$

for each $x, y, z \in X$ and $t>0$. Observe that all the terms on the right-hand side of the above inequality tend to 1 as $n \rightarrow \infty$, since $1 / 2<q<1$. Hence, together with the similar argument after (15), we can say that $\operatorname{DF}(x, y, z)=0$, for all $x, y, z \in X$. Recall, in Case 1, the inequality (6) follows from (14). By the same reasoning, we get (6) from (26) in this case. Now to prove the uniqueness of $F$, let $F^{\prime}$ be another general quadratic function satisfying (6). Then, together with (N4), (6), and (20), we have

$$
\begin{aligned}
N^{\prime}\left(F(x)-F^{\prime}(x), t\right) & \\
= & N^{\prime}\left(J_{n} F(x)-J_{n} F^{\prime}(x), t\right), \\
\geq \min \{ & N^{\prime}\left(J_{n} F(x)-J_{n} f(x), \frac{t}{2}\right), \\
& \left.N^{\prime}\left(J_{n} f(x)-J_{n} F^{\prime}(x), \frac{t}{2}\right)\right\}, \\
\geq \min \{ & N^{\prime}\left(\frac{(F-f)\left(2^{n} x\right)}{2 \cdot 4^{n}}, \frac{t}{8}\right),\left(\frac{\left(f-F^{\prime}\right)\left(2^{n} x\right)}{2 \cdot \frac{t}{8}}\right), \\
& N^{\prime}\left(\frac{(F-f)\left(-2^{n} x\right)}{2 \cdot 4^{n}}, \frac{t}{8}\right), \\
& N^{\prime}\left(2^{n-1}\left((F-f)\left(\frac{-x}{2^{n}}\right)\right), \frac{t}{8}\right), \\
& N^{\prime}\left(\frac{\left(f-F^{\prime}\right)\left(-2^{n} x\right)}{2 \cdot 4^{n}}, \frac{t}{8}\right), \\
& N^{\prime}\left(2^{n-1}\left((F-f)\left(\frac{x}{2^{n}}\right)\right), \frac{t}{8}\right),
\end{aligned}
$$




$$
\begin{aligned}
& \geq \min \left\{\operatorname { s u p } _ { t ^ { \prime } < t } N \left(x, 2^{(2 q-1) n-2 q}\right.\right. \\
&\left.\quad \times\left(\frac{2\left(4-2^{p}\right)\left(2^{p}-2\right) 2^{p}}{-2 \cdot 8^{p}+3 \cdot 4^{p}+34 \cdot 2^{p}-20}\right)^{q} t^{\prime q}\right), \\
& \sup _{t^{\prime}<t} N\left(x, 2^{(1-q) n-2 q}\right. \\
&\left.\left.\times\left(\frac{2\left(4-2^{p}\right)\left(2^{p}-2\right) 2^{p}}{-2 \cdot 8^{p}+3 \cdot 4^{p}+34 \cdot 2^{p}-20}\right)^{q} t^{\prime q}\right)\right\},
\end{aligned}
$$

for all $x \in X$ and $n \in \mathbb{N}$. Since $\lim _{n \rightarrow \infty} 2^{(2 q-1) n-2 q}=$ $\lim _{n \rightarrow \infty} 2^{(1-q) n-2 q}=\infty$; in this case, both terms on the right hand side of the above inequality tend to 1 as $n \rightarrow \infty$ by (N5). This implies that $N^{\prime}\left(F(x)-F^{\prime}(x), t\right)=1$, and so $F(x)=F^{\prime}(x)$ for all $x \in X$ by (N2).

Case 3. Finally, we take $0<q<1 / 2$ and define $J_{n} f: X \rightarrow Y$ by

$$
\begin{aligned}
J_{n} f(x)= & \frac{4^{n}}{2}\left(f\left(2^{-n} x\right)+f\left(-2^{-n} x\right)-2 f(0)\right) \\
& +2^{n-1}\left(f\left(\frac{x}{2^{n}}\right)-f\left(-\frac{x}{2^{n}}\right)\right)+f(0),
\end{aligned}
$$

for all $x \in X$. Then we have $J_{0} f(x)=f(x), J_{j} f(0)=f(0)$, and

$$
\begin{aligned}
J_{j} f(x) & -J_{j+1} f(x) \\
= & \frac{4^{j}}{2}\left(D f\left(\frac{x}{2^{j+2}}, \frac{x}{2^{j+2}}, \frac{x}{2^{j+1}}\right)+D f\left(\frac{x}{2^{j+2}}, \frac{x}{2^{j+2}}, \frac{x}{2^{j+2}}\right)\right.
\end{aligned}
$$

$$
\begin{aligned}
& \left.+D f\left(\frac{-x}{2^{j+2}}, \frac{-x}{2^{j+2}}, \frac{-x}{2^{j+1}}\right)+D f\left(\frac{-x}{2^{j+2}}, \frac{-x}{2^{j+2}}, \frac{-x}{2^{j+2}}\right)\right) \\
& -2^{j-1}\left(D f\left(\frac{x}{2^{j}}, \frac{x}{2^{j+1}}, \frac{x}{2^{j+1}}\right)-D f\left(\frac{x}{2^{j+1}}, \frac{x}{2^{j}}, \frac{x}{2^{j+1}}\right)\right.
\end{aligned}
$$

$$
\left.+D f\left(\frac{x}{2^{j+1}}, \frac{x}{2^{j+1}}, \frac{x}{2^{j+1}}\right)\right),
$$

$$
\begin{aligned}
& N^{\prime}\left(J_{m} f(x)-J_{n+m} f(x), \sum_{j=m}^{n+m-1}\left(\frac{\left(5+2^{p}\right) 4^{j}}{2^{(j+2) p}}+\frac{\left(7+2 \cdot 2^{p}\right) 2^{j}}{2 \cdot 2^{(j+1) p}}\right) t^{p}\right) \\
& \geq \min \bigcup_{j=m}^{n+m-1}\left\{N^{\prime}\left(\frac{4^{j}}{2} D f\left(\frac{x}{2^{j+2}}, \frac{x}{2^{j+2}}, \frac{x}{2^{j+1}}\right), \frac{4^{j}\left(2+2^{p}\right)}{2 \cdot 2^{(j+2) p}} t^{p}\right),\right. \\
& N^{\prime}\left(\frac{4^{j}}{2} D f\left(\frac{x}{2^{j+2}}, \frac{x}{2^{j+2}}, \frac{x}{2^{j+2}}\right), \frac{3 \cdot 4^{j} t^{p}}{2 \cdot 2^{(j+2) p}}\right), \\
& N^{\prime}\left(\frac{4^{j}}{2} D f\left(\frac{-x}{2^{j+2}}, \frac{-x}{2^{j+2}}, \frac{-x}{2^{j+1}}\right), \frac{4^{j}\left(2+2^{p}\right)}{2 \cdot 2^{(j+2) p}} t^{p}\right), \\
& N^{\prime}\left(\frac{4^{j}}{2} D f\left(\frac{-x}{2^{j+2}}, \frac{-x}{2^{j+2}}, \frac{-x}{2^{j+2}}\right), \frac{3 \cdot 4^{j} t^{p}}{2 \cdot 2^{(j+2) p}}\right), \\
& N^{\prime}\left(-2^{j-1} D f\left(\frac{x}{2^{j}}, \frac{x}{2^{j+1}}, \frac{x}{2^{j+1}}\right), \frac{2^{j-1}\left(2+2^{p}\right) t^{p}}{2^{(j+1) p}}\right), \\
& N^{\prime}\left(2^{j-1} D f\left(\frac{x}{2^{j+1}}, \frac{x}{2^{j}}, \frac{x}{2^{j+1}}\right), \frac{2^{j-1}\left(2+2^{p}\right) t^{p}}{2^{(j+1) p}}\right), \\
& \left.N^{\prime}\left(-2^{j-1} D f\left(\frac{x}{2^{j+1}}, \frac{x}{2^{j+1}}, \frac{x}{2^{j+1}}\right), \frac{3 \cdot 2^{j-1} t^{p}}{2^{(j+1) p}}\right)\right\},
\end{aligned}
$$




$$
\begin{aligned}
& \geq \min \bigcup_{j=m}^{n+m-1}\left\{N\left(\frac{x}{2^{j}}, \frac{t}{2^{j}}\right), N\left(\frac{x}{2^{j+1}}, \frac{t}{2^{j+1}}\right), N\left(\frac{x}{2^{j+2}}, \frac{t}{2^{j+2}}\right)\right\}, \\
& =N(x, t),
\end{aligned}
$$

for all $x \in X, j \geq 0$, and $t>0$. Similar to the previous cases, it leads us to define the function $F: X \rightarrow Y$ by $F(x):=$ $N^{\prime}-\lim _{n \rightarrow \infty} J_{n} f(x)$. Putting $m=0$ in the above inequality, we have

$$
\begin{aligned}
& N^{\prime}\left(f(x)-J_{n} f(x), t\right) \\
& \quad \geq N\left(x, t^{q} /\left(\sum_{j=0}^{n-1}\left(\frac{\left(5+2^{p}\right) 4^{j}}{2^{(j+2) p}}+\frac{\left(7+2 \cdot 2^{p}\right) 2^{j}}{2 \cdot 2^{(j+1) p}}\right)\right)^{q}\right),
\end{aligned}
$$

for all $x \in X$ and $t>0$. Notice that

$$
\begin{gathered}
N^{\prime}\left(D J_{n} f(x, y, z), \frac{3 t}{4}\right) \\
\geq \min \left\{N^{\prime}\left(\frac{4^{n}}{2} D f\left(\frac{x}{2^{n}}, \frac{y}{2^{n}}, \frac{z}{2^{n}}\right), \frac{3 t}{16}\right),\right. \\
N^{\prime}\left(\frac{4^{n}}{2} D f\left(\frac{-x}{2^{n}}, \frac{-y}{2^{n}}, \frac{-z}{2^{n}}\right), \frac{3 t}{16}\right), \\
N^{\prime}\left(2^{n-1} D f\left(\frac{x}{2^{n}}, \frac{y}{2^{n}}, \frac{z}{2^{n}}\right), \frac{3 t}{16}\right), \\
\left.N^{\prime}\left(2^{n-1} D f\left(\frac{-x}{2^{n}}, \frac{-y}{2^{n}}, \frac{-z}{2^{n}}\right), \frac{3 t}{16}\right)\right\}, \\
\geq \min \left\{N\left(x, 2^{(1-2 q) n-3 q} t^{q}\right), N\left(y, 2^{(1-2 q) n-3 q} t^{q}\right),\right. \\
\left.N\left(z, 2^{(1-2 q) n-3 q} t^{q}\right)\right\},
\end{gathered}
$$

for all $x, y, z \in X$ and $t>0$. Since $0<q<1 / 2$, all terms on the right hand side tend to 1 as $n \rightarrow \infty$, which implies that the last term of (15) tends to 1 as $n \rightarrow \infty$. Therefore, we can say that $D F \equiv 0$. Moreover, using the similar argument after (15) in Case 1, we get the inequality (6) from (32) in this case. To prove the uniqueness of $F$, let $F^{\prime}: X \rightarrow Y$ be another general quadratic function satisfying (6). Then by (20), we get

$$
\begin{aligned}
& N^{\prime}\left(F(x)-F^{\prime}(x), t\right) \\
& \geq \min \left\{N^{\prime}\left(J_{n} F(x)-J_{n} f(x), \frac{t}{2}\right),\right. \\
& \left.\quad N^{\prime}\left(J_{n} f(x)-J_{n} F^{\prime}(x), \frac{t}{2}\right)\right\},
\end{aligned}
$$

$$
\begin{aligned}
& \geq \min \{ N^{\prime}\left(\frac{4^{n}}{2}\left((F-f)\left(\frac{x}{2^{n}}\right)\right), \frac{t}{8}\right), \\
& \frac{4^{n}}{2}\left(\left(f-F^{\prime}\right)\left(\frac{x}{2^{n}}\right), \frac{t}{8}\right), \\
& N^{\prime}\left(\frac{4^{n}}{2}\left((F-f)\left(-\frac{x}{2^{n}}\right)\right), \frac{t}{8}\right), \\
& N^{\prime}\left(\frac{4^{n}}{2}\left(\left(f-F^{\prime}\right)\left(-\frac{x}{2^{n}}\right)\right), \frac{t}{8}\right), \\
& N^{\prime}\left(2^{n-1}\left((F-f)\left(\frac{x}{2^{n}}\right)\right), \frac{t}{8}\right), \\
& N^{\prime}\left(2^{n-1}\left(\left(f-F^{\prime}\right)\left(\frac{x}{2^{n}}\right)\right), \frac{t}{8}\right), \\
& N^{\prime}\left(2^{n-1}\left((F-f)\left(\frac{-x}{2^{n}}\right)\right), \frac{t}{8}\right), \\
&\left.N^{\prime}\left(2^{n-1}\left(\left(f-F^{\prime}\right)\left(\frac{-x}{2^{n}}\right)\right), \frac{t}{8}\right)\right\}, \\
&\left.\times\left(\frac{2\left(2^{p}-4\right)\left(2^{p}-2\right) 2^{p}}{2 \cdot 8 p+4 p-22 \cdot 2^{p}-20}\right)^{q} t^{\prime q}\right), \\
& \geq \sup _{t^{\prime}<t} N\left(x, 2^{(1-2 q) n-2 q}\right.
\end{aligned}
$$

for all $x \in X$ and $n \in \mathbb{N}$. Observe that, for $0<q=1 / p<1 / 2$, the last term tends to 1 as $n \rightarrow \infty$ by (N5). This implies that $N^{\prime}\left(F(x)-F^{\prime}(x), t\right)=1$ and $F(x)=F^{\prime}(x)$, for all $x \in X$ by (N2).

We can use Theorem 1 to get a classical result in the framework of normed spaces. Let $(X,\|\cdot\|)$ be a normed linear space. Then we can define a fuzzy norm $N_{X}$ on $X$ by following

$$
N_{X}(x, t)= \begin{cases}0 & \text { if } t \leq\|x\|, \\ 1 & \text { if } t>\|x\|,\end{cases}
$$

where $x \in X$ and $t \in \mathbb{R}[21]$. Suppose that $f: X \rightarrow Y$ is a function into a Banach space $(Y,|\|\cdot\||)$ such that

$$
\|\mid D f(x, y, z)\|\|\leq\| x\left\|^{p}+\right\| y\left\|^{p}+\right\| z \|^{p},
$$

for all $x, y, z \in X$, where $p>0$ and $p \neq 1,2$. Let $N_{Y}$ be a fuzzy norm on $Y$. Then we get

$$
\begin{aligned}
N_{Y}(D f(x, y, z), r+s+t) \\
\quad= \begin{cases}0 & \text { if } r+s+t \leq\|\mid D f(x, y, z)\|, \\
1 & \text { if } r+s+t>\|\mid D f(x, y, z)\|,\end{cases}
\end{aligned}
$$


for all $x, y, z \in X$ and $r, s, t \in \mathbb{R}$. Consider the case $N_{Y}(D f(x, y, z), r+s+t)=0$. This implies that

$$
\|x\|^{p}+\|y\|^{p}+\|z\|^{p} \geq|D f(x, y, z)| \geq r+s+t,
$$

and so either $\|x\|^{p} \geq r$ or $\|y\|^{p} \geq s$ or $\|z\|^{p} \geq t$ in this case. Hence, for $q=1 / p$, we have

$$
\min \left\{N_{X}\left(x, r^{q}\right), N_{X}\left(y, s^{q}\right), N_{X}\left(z, t^{q}\right)\right\}=0,
$$

for all $x, y, z \in X$ and $r, s, t>0$. Therefore, in every case, the inequality

$$
\begin{aligned}
& N_{Y}(D f(x, y, z), r+s+t) \\
& \quad \geq \min \left\{N_{X}\left(x, r^{q}\right), N_{X}\left(y, s^{q}\right), N_{X}\left(z, t^{q}\right)\right\},
\end{aligned}
$$

holds. It means that $f$ is a fuzzy $q$-almost general quadratic function, and by Theorem 1, we get the following stability result.

Corollary 1. Let $(X,\|\cdot\|)$ be a normed linear space, and let $(Y,\||\cdot|\|)$ be a Banach space. If

$$
\||D f(x, y, z)|\| \leq\|x\|^{p}+\|y\|^{p}+\|z\|^{p}
$$

for all $x, y, z \in X$, where $p>0$ and $p \neq 1,2$, then there is a unique general quadratic function $F: X \rightarrow Y$ such that

$$
\||F(x)-f(x)|\| \leq\left(\frac{5+2^{p}}{2^{p} \mid 4-2^{p \mid}}+\frac{2 \cdot 2^{p}+7}{2 \mid 2-2^{p \mid}}\right)\|x\|^{p},
$$

for all $x \in X$.

Remark 1. Consider a function $f: X \rightarrow Y$ satisfying (5) for all $x, y, z \in X \backslash\{0\}$ and a real number $q<0$. Take any $t>0$. If we choose a real number $s$ with $0<3 s<t$, then we have

$$
\begin{aligned}
N^{\prime}(D f(x, y, z), t) & \geq N^{\prime}(D f(x, y, z), 3 s) \\
& \geq \min \left\{N\left(x, s^{q}\right), N\left(y, s^{q}\right), N\left(z, s^{q}\right)\right\},
\end{aligned}
$$

for all $x, y, z \in X \backslash\{0\}$. Since $q<0$, we have $\lim _{s \rightarrow 0^{+}} q=\infty$. This implies that

$$
\lim _{s \rightarrow 0^{+}} N\left(x, s^{q}\right)=\lim _{s \rightarrow 0^{+}} N\left(y, s^{q}\right)=\lim _{s \rightarrow 0^{+}} N\left(z, s^{q}\right)=1,
$$

and so

$$
N^{\prime}(D f(x, y, z), t)=1,
$$

for all $t>0$ and $x, y, z \in X \backslash\{0\}$. By (N2), it allows us to get $D f(x, y, z)=0$, for all $x, y, z \in X \backslash\{0\}$.

\section{References}

[1] S. M. Ulam, A Collection of Mathematical Problems, Interscience, New York, NY, USA, 1968.

[2] D. H. Hyers, "On the stability of the linear functional equation," Proceedings of the National Academy of Sciences of the United States of America, vol. 27, pp. 222-224, 1941.
[3] T. Aoki, "On the stability of the linear transformation in Banach spaces," Journal of the Mathematical Society of Japan, vol. 2, pp. 64-66, 1950.

[4] Th. M. Rassias, "On the stability of the linear mapping in Banach spaces," Proceedings of the American Mathematical Society, vol. 72, no. 2, pp. 297-300, 1978.

[5] M. Adam and S. Czerwik, "On the stability of the quadratic functional equation in topological spaces," Banach Journal of Mathematical Analysis, vol. 1, no. 2, pp. 245-251, 2007.

[6] St. Czerwik, "On the stability of the quadratic mapping in normed spaces," Abhandlungen aus dem Mathematischen Seminar der Universität Hamburg, vol. 62, pp. 59-64, 1992.

[7] P. Găvruţa, "A generalization of the Hyers-Ulam-Rassias stability of approximately additive mappings," Journal of Mathematical Analysis and Applications, vol. 184, no. 3, pp. 431-436, 1994.

[8] G. H. Kim, "On the stability of functional equations with square-symmetric operation," Mathematical Inequalities \& Applications, vol. 4, no. 2, pp. 257-266, 2001.

[9] Y.-H. Lee, "On the Hyers-Ulam-Rassias stability of the generalized polynomial function of degree 2," The Journal of Chungcheong Mathematical Society, vol. 22, pp. 201-209, 2009.

[10] Y.-H. Lee, "On the stability of the monomial functional equation," Bulletin of the Korean Mathematical Society, vol. 45, no. 2, pp. 397-403, 2008.

[11] Y.-H. Lee and K.-W. Jun, "A generalization of the Hyers-UlamRassias stability of Jensen's equation," Journal of Mathematical Analysis and Applications, vol. 238, no. 1, pp. 305-315, 1999.

[12] Y.-H. Lee and K.-W. Jun, "A generalization of the HyersUlam-Rassias stability of the Pexider equation," Journal of Mathematical Analysis and Applications, vol. 246, no. 2, pp. 627-638, 2000.

[13] Y.-H. Lee and K.-W. Jun, "On the stability of approximately additive mappings," Proceedings of the American Mathematical Society, vol. 128, no. 5, pp. 1361-1369, 2000.

[14] A. K. Mirmostafaee, M. Mirzavaziri, and M. S. Moslehian, "Fuzzy stability of the Jensen functional equation," Fuzzy Sets and Systems, vol. 159, no. 6, pp. 730-738, 2008.

[15] F. Skof, "Local properties and approximation of operators," Rendiconti del Seminario Matematico e Fisico di Milano, vol. 53, pp. 113-129, 1983.

[16] A. K. Katsaras, "Fuzzy topological vector spaces. II," Fuzzy Sets and Systems, vol. 12, no. 2, pp. 143-154, 1984.

[17] T. Bag and S. K. Samanta, "Finite dimensional fuzzy normed linear spaces," Journal of Fuzzy Mathematics, vol. 11, no. 3, pp. 687-705, 2003.

[18] S. C. Cheng and J. N. Mordeson, "Fuzzy linear operators and fuzzy normed linear spaces," Bulletin of the Calcutta Mathematical Society, vol. 86, no. 5, pp. 429-436, 1994.

[19] I. Kramosil and J. Michálek, "Fuzzy metrics and statistical metric spaces," Kybernetika, vol. 11, no. 5, pp. 336-344, 1975.

[20] A. K. Mirmostafaee and M. S. Moslehian, "Fuzzy versions of Hyers-Ulam-Rassias theorem," Fuzzy Sets and Systems, vol. 159, no. 6, pp. 720-729, 2008.

[21] A. K. Mirmostafaee and M. S. Moslehian, "Fuzzy almost quadratic functions," Results in Mathematics, vol. 52, no. 1-2, pp. 161-177, 2008.

[22] H.-M. Kim, "Hyers-Ulam stability of a general quadratic functional equation," Publications de l'Institut Mathématique, vol. 73(87), pp. 129-137, 2003.

[23] K.-W. Jun and H.-M. Kim, "The generalized Hyers-Ulam stability of a general quadratic functional equation," Journal of Applied Mathematics \& Computing, vol. 15, no. 3, pp. 377392, 2004. 

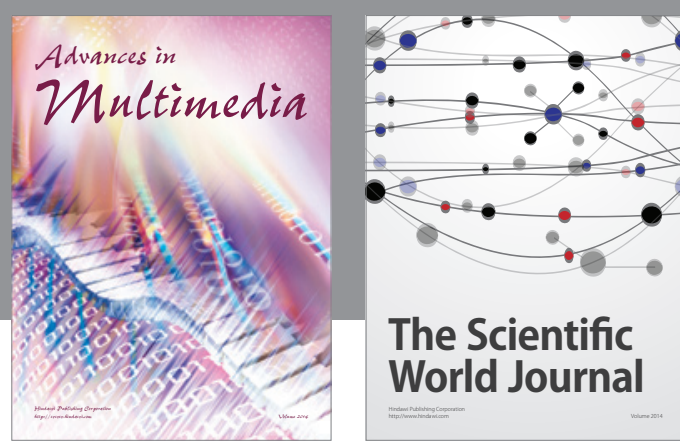

The Scientific World Journal
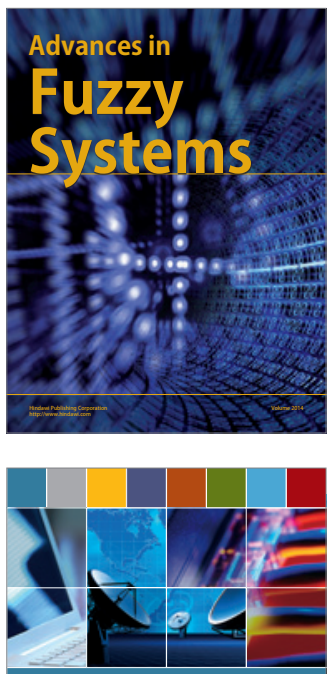

Computer Networks and Communications
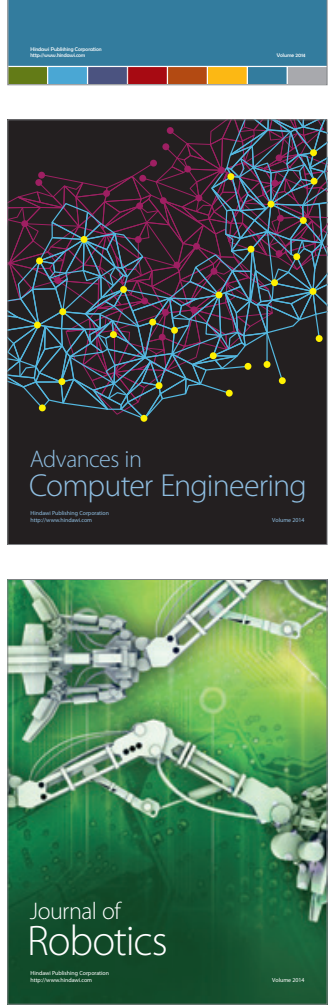
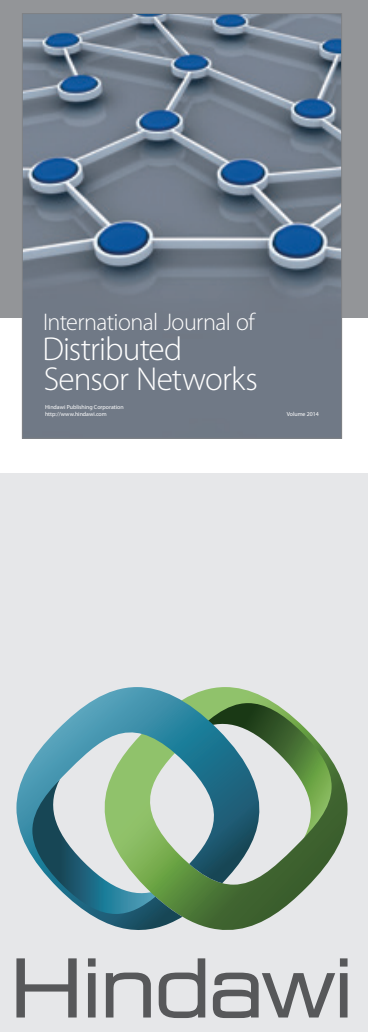

Submit your manuscripts at

http://www.hindawi.com
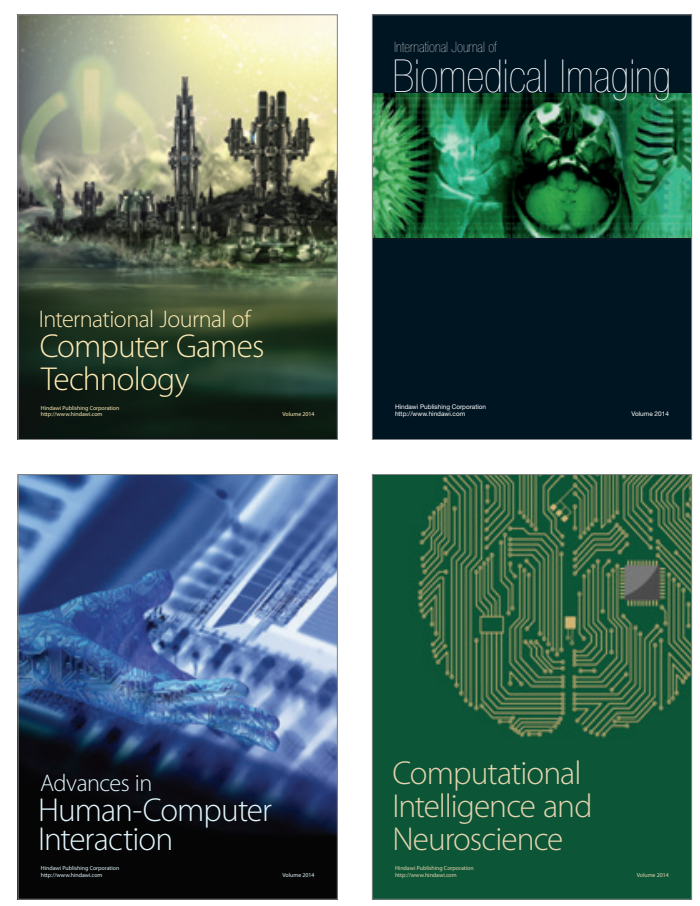
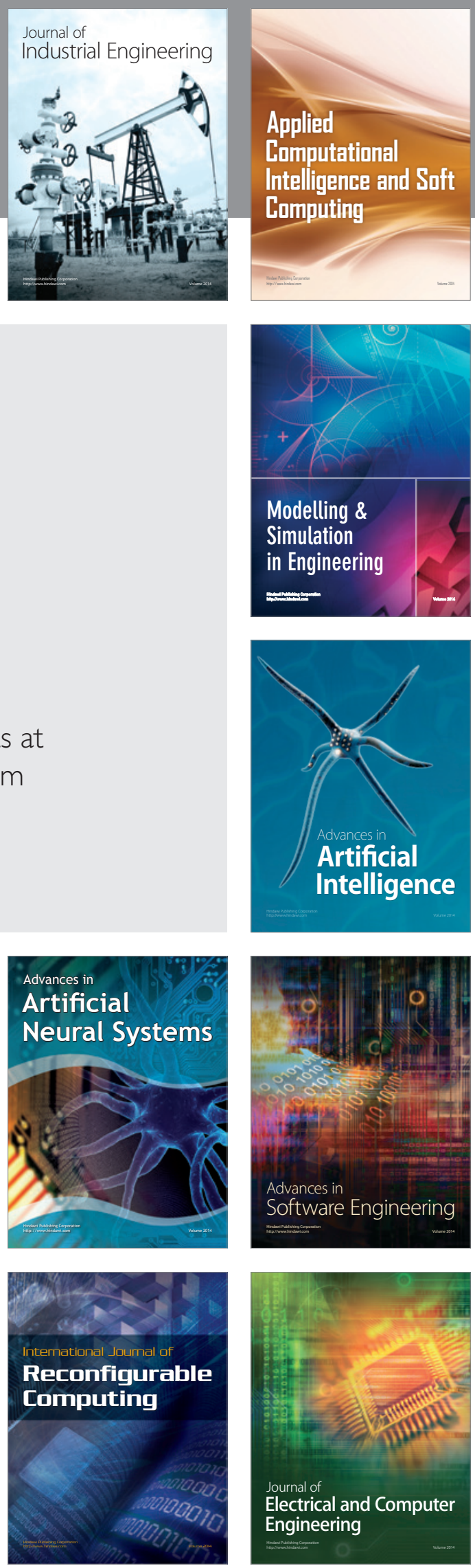